\title{
The Metacognitions about Symptoms Control Scale: Development and concurrent validity
}

\author{
Word count: 4,830 (all sections included) \\ Full Length Paper
}

Bruce A. Fernie

King’s College London, Institute of Psychiatry, Department of Psychology, London, UK

CASCAID, South London \& Maudsley NHS Foundation Trust, London, UK

Lorraine Maher-Edwards

Chelsea and Westminster NHS Foundation Trust, London, UK

Gabrielle Murphy

Fatigue Service, Royal Free London NHS Foundation Trust, UK

Ana V. Nikčević

Faculty of Arts and Social Sciences, Kingston University,

Kingston upon Thames, UK

Marcantonio M. Spada

London South Bank University, London, UK

\section{Revision 2}

\section{April 2014}

This is the peer reviewed version of the following article: Fernie, B. A., Maher-Edwards, L., Murphy, G., Nikčević, A. V., and Spada, M. M. (2015), The Metacognitions about Symptoms Control Scale: Development and Concurrent Validity. Clin. Psychol. Psychother., 22, 443449., which has been published in final form at http://dx.doi.org/10.1002/cpp.1906. This article may be used for non-commercial purposes in accordance with Wiley Terms and Conditions for Self-Archiving."

\section{Acknowledgements}

Author Bruce A. Fernie receives salary support from the National Institute for Health Research (NIHR) Mental Health Biomedical Research Centre and Dementia Research Unit at South London and Maudsley NHS Foundation Trust and King's College London. The views expressed are those of the author and not necessarily those of the NHS, the NIHR or the Department of Health. 


\title{
Author Notes
}

Correspondence should be addressed to: Marcantonio M. Spada, Department of Psychology, Faculty of Arts and Human Sciences, London South Bank University, United Kingdom. Tel. +44 (0)20 7815 5760, e-mail spadam@1sbu.ac.uk.

\begin{abstract}
Objective: This paper presents the development and preliminary validation of a self-report instrument designed to measure metacognitions pertaining to symptoms control in the form of: (1) symptoms focusing; and (2) symptoms conceptual thinking. Methods: 124 patients (95 female and 29 male) presenting with Chronic Fatigue Syndrome (CFS) contributed data to the study to test the structure and psychometric properties of the Metacognitions about Symptoms Control Scale (MaSCS). Results: A principal components factor analysis indicated that a two-factor solution best fitted the data. The factors were labelled positive and negative metacognitions about symptoms control. Further analyses revealed that both factors had good internal consistency. Correlation analyses established preliminary concurrent validity, indicating that both positive and negative metacognitions about symptoms control were significantly associated with levels of fatigue in CFS. Regression analysis revealed that positive and negative metacognitions about symptoms control significantly predicted fatigue severity when controlling for anxiety and depression. Conclusions: The newly developed instrument may help future research that examines the role of metacognitions in CFS, as well as aiding clinical assessment and case formulation.
\end{abstract}

\section{Key Practitioner Message}

The MaSCS is a useful first instrument to assess metacognitions in CFS.

The MaSCS may help to deepen our understanding of symptoms control (symptoms focusing and conceptual thinking about symptoms) in the experience of CFS symptoms.

Assessing and conceptualising symptoms control through the MaSCS may aid treatment of CFS. 
Key words: chronic fatigue syndrome; metacognitions; self-report instrument; symptoms focusing; symptoms conceptual thinking.

\section{Introduction}

Individuals with Chronic Fatigue Syndrome (CFS) often present with a myriad of symptoms (Nisenbaum, Reyes, Unger, \& Reeves, 2004), chiefly characterised by fatigue (Sharpe et al., 1991). Flu-like symptoms without the presence of fever are often reported (Komaroff et al., 1996), alongside cognitive deficits and neurological symptoms (Friedberg, Dechene, McKenzie Ii, \& Fontanetta, 2000).

A series of systematic reviews have suggested that Cognitive Behaviour Therapy (CBT; Price, Mitchell, Tidy, \& Hunot, 2008) and Graded Exercise Therapy (GET; Edmonds, McGuire, \& Price, 2004) are at least moderately effective treatments for CFS. More recently, these reviews have been supported by the publication of the PACE trial, a large multi-centred randomised trial that compared the effectiveness of adding adaptive pacing therapy (APT), GET, or CBT to standardised medical care (SMC; White et al., 2011). This study found that CBT and GET were moderately effective treatments for CFS, and that there was no difference between APT and SMC, and SMC alone. CBT and GET remain the two treatments recommended by the National Institute of Clinical Excellence guidelines (2007) for mild and moderate categories of CFS; however, recovery rates in CFS occupy a broad range, depending on the definition of recovery used. Knoop, Bleijenberg, Gielissen, van der Meer, and White (2007) suggest that between 23\% and 69\% of CFS patients make a full recovery following a course of CBT. Whilst this finding is encouraging, it suggests that it may be beneficial to examine refining CBT protocols to enhance treatment outcomes.

Traditional CBT protocols for CFS attempt to address factors involved in the maintenance of the condition such as all-or-nothing patterns of behaviour, maladaptive coping strategies, and unhelpful beliefs about symptoms and activity (White, Sharpe, Chalder, DeCesare, \& Walwyn, 
2007). However, recent research has implicated cognitive processes in CFS that offer themselves as potential new targets for intervention. For example conceptual thinking, such as self-reported health-related worry and preoccupation with health threats (Aggarwal, McBeth, Zakrzewska, Lunt, \& Macfarlane, 2006), has been found in CFS, as well as attentional biases for processing healththreat information (Hou, Moss-Morris, Bradley, Peveler, \& Mogg, 2008).

Metacognitions can be defined as “stable knowledge or beliefs about one’s own cognitive system, and knowledge about factors that affect the functioning of the system; the regulation and awareness of the current state of cognition, and appraisal of the significance of thought and memories” (p. 302; Wells, 1995). Metacognitions are divided into two broad sets (Wells \& Matthews, 1994; Wells, 2000): (1) positive metacognitions about control strategies that impact on inner events such as "Worry will help me get things sorted out in my mind" or "If I ruminate I will find a solution”; and (2) negative metacognitions concerning the significance, controllability and danger of inner events, such as "It is bad to have sensation X" or "My worry is out of control". Metacognitions are believed to play a fundamental role in activating control strategies (such as attention to threat, conceptual thinking and thought suppression) in response to inner events (such as thoughts, emotional states or physical symptoms) that result in the exacerbation and maintenance of psychological distress. In support of this view, metacognitions have been found to be associated with a wide array of psychological and behavioural problems (for a full review, see Wells, 2008; Wells, 2013). Related research by Dimaggio and colleagues (2007) and Lysaker and colleagues (2014) has also highlighted the importance of an awareness of one's own mental state and the ability to recognize the mental states of others in personality disorder. This is of particular relevance in studying metacognition in CFS because of the high prevalence of personality disorders found in CFS (e.g. Henderson \& Tannock, 2004).

Recent research on CFS has suggested that metacognitions about the need to control thoughts and cognitive confidence predict symptom severity in CFS when controlling for negative emotion (Lorraine Maher-Edwards, Fernie, Murphy, Wells, \& Spada, 2011). A later study on CFS 
patients, by Maher-Edwards and colleagues (Maher-Edwards, Fernie, Murphy, Nikčević, \& Spada, 2012), identified positive metacognitions about symptoms focusing and conceptual thinking about symptoms. The authors argued that such beliefs may activate these conceptual processes in response to CFS symptoms. Negative metacognitions about the drawbacks (worsening of negative affect) and uncontrollability of symptoms focusing and conceptual thinking about symptoms were also identified. The authors argued that these beliefs could play a role both in worsening the experience of fatigue because of worsening negative affect (Sohl \& Friedberg, 2008) as well as result in the perseveration of fatigue because attempts at terminating conceptual processing would become less likely.

The current study builds on these findings by presenting the development and preliminary validation of an instrument designed to assess CFS-specific metacognitions about symptoms control in the form of symptoms focusing and conceptual thinking about symptoms. The instrument was built from items derived from the aforementioned studies, clinical experience, and theory. We hypothesized that this newly developed instrument would have a significant association with the severity of CFS symptoms and that this relationship would be maintained when controlling for anxiety and depression.

\section{Methods}

\subsection{Participants}

One hundred and twenty four patients (95 female and 29 male) referred to the Fatigue Service at the Royal Free London NHS Foundation Trust, UK, were recruited for the purposes of this study. All patients were given a formal diagnosis of CFS according to the Oxford Criteria (Sharpe et al., 1991). The self-reported mean time since the onset of symptoms was 9.1 years (range 1-45). Prior to diagnosis, all other known causes for fatigue were excluded on history, examination and by investigation. The latter included blood and urine testing for most of the common, and some of the less common, causes of fatigue such as anaemia, diabetes, autoimmune conditions such as coeliac disease, common variable immunodeficiency, and thyroid dysfunction as well as screen for 
infection and inflammation. A full physical examination was also conducted, including standing and lying blood pressure measurements, specifically to exclude markers of quiescent conditions that may not show on blood tests and orthostatic hypotension and postural orthostatic tachycardia syndrome.

Additional inclusion criteria were: (1) 18 years of age or above; (2) consenting to the study; (3) understanding spoken and written English; (4) the absence of a diagnosis of major depressive disorder or psychosis. The mean age of the sample was 41.7 years (range 18-70). Forty three patients had previously undertaken a course of CBT, four patients had undertaken a course of GET, 24 patients had undertaken an alternative therapy (defined as a therapy not recommended in the NICE guidelines for CFS), and 11 patients had undertaken both an alternative therapy together with either or both CBT and GET.

\subsection{Self-Report Instruments}

\subsubsection{Chalder Fatigue Questionnaire (CFQ; Chalder et al., 1993)}

The CFQ consists of 14 items assessing levels of mental (8 items; e.g. "Do you need to rest more?”) and physical (6 items; e.g. Do you have problems with tiredness?”) fatigue over the previous month. Higher scores indicate higher levels of mental and physical fatigue. The CFQ possesses good psychometric properties (Chalder et al., 1993)and is recommended for use in the Adult Minimum Dataset for assessing severity of CFS (NICE, 2007). The instrument utilizes a fourpoint Likert-type response form. Items responded to as 'better than usual' and 'no worse than usual' are sometimes scored as 0 , and items responded to as 'worse than usual' and 'much worse than usual' are scored as 1 . The present study scored responses as 1, 2, 3, or 4 respectively, with higher scores representing more fatigue. This scoring approach was taken to heighten the sensitivity of the instrument, retaining its original psychometric properties, for the purpose of elucidating the relationship between CFS symptoms and the newly developed instrument.

\subsubsection{RAND Short Form 36 Physical Functioning (SF-36 PF; Ware \& Sherbourne, 1992)}


The RAND SF-36 PF consist of 10 items assessing daily activities such as climbing stairs, lifting or carrying groceries, walking, and bathing. It is the Physical Functioning factor of the RAND Short Form 36 Health Questionnaire that assesses function in different areas such as general health, social functioning, mental health and limitations in role activities because of physical or emotional problems (Ware \& Sherbourne, 1992). Participants indicate how much their health limits them in these activities, responding to individual items by choosing between "A lot”, "A little” or “Not at all”. Higher scores indicate higher levels of physical functioning. The RAND SF-36 PF is a widely used, reliable and valid instrument with population norms and normative data for a variety of medical conditions (Ware \& Kosinski, 2001).

\subsubsection{Depression Anxiety Stress Scale 21 (DASS-21; Lovibond \& Lovibond, 1995)}

The DASS-21 consists of 21 items assessing levels of depression (e.g. "I felt that I had nothing to look forward to”), anxiety (e.g. “I felt scared without any good reason”) and stress (e.g. “I was intolerant of anything that kept me from getting on with what I was doing”). Higher scores indicate higher levels of anxiety, depression and stress. The DASS-21 has been reported to have adequate psychometric properties(Henry \& Crawford, 2005), with the factors of the full version having been shown to correlate with validated measures of anxiety and depression (Crawford \& Henry, 2003). This study utilized the anxiety and depression factors.

\subsubsection{Metacognitions about Symptoms Control Scale (MaSCS)}

The MaSCS items were derived from responses to an earlier metacognitive profiling study in CFS (Maher-Edwards et al., 2012), from the authors' clinical experience, and from deductions based on theory. The raw MaSCS consisted of 19 items and utilised a four-point Likert-type response format, with the options "Do not agree”, “Agree Slightly”, “Agree moderately”, and “Agree strongly”. The items were presented with the following preamble:

This scale is concerned with how people with CFS/ME experience and cope with their symptoms. Listed below are a number of beliefs that people have expressed. Please read each item 
and say how much you generally agree with it by circling the one appropriate number. There are no right or wrong answers.

\subsection{Procedure}

Ethics approval was obtained from the local research ethics committee. Patients received the study pack through the post. They were informed that the study aimed to investigate how people with CFS think about and experience symptoms. Patients were informed that participation was voluntary, that confidentiality would be protected, and gave informed consent to participate in the study.

\subsection{Data Analysis}

A principal components analysis was conducted on the data. Internal consistency was assessed by calculating Cronbach’s Alphas for all factors and by calculating alphas-if-item-deleted. The data from was also assessed for normality with Kolmogorov-Smirnov tests. Concurrent validity was assessed by computing correlations between the factors of the MaSCS and factors of the CFQ and SF-36 PF. A regression analysis was conducted to assess the relationship between the MaSCS and CFQ when controlling for anxiety and depression.

\section{Results}

\subsection{Principal Components Analysis of the MaSCS}

A principal components analysis was conducted on the original items of the MaSCS (19 items). Scree tests for the raw instrument suggested a two-factor solution with Eigen values of 6.0 and 3.9. Indicators of the latent variables were assessed using a Promax rotation with a Kappa of four. This oblique rotation enabled us to assess the correlation between factors $(r=.20)$. The two factors combined accounted for 51.9\% of the variance.

Item selection was based on the following criteria: (1) if an item loaded less than .4 on a factor it was discarded; (2) if an item loaded more than .4 on both factors it was discarded; and (3) 
if an item loaded 0.4 or greater on a factors but it's loading on the other factor was within .2, it was discarded.

The revised instrument retained 17 items, with the distribution of nine items on one factor and eight on the other. For the revised instrument, the two-factor solution accounted for $55.3 \%$ of the variance (with Eigen values of 5.6 and 3.9) and the estimated correlation between the factors was .17. Table 1 shows the factor loadings for the revised instrument. The first factor of the instrument was named positive metacognitions about symptoms control and the second factor negative metacognitions about symptoms control as these appeared to reflect their content.

\subsection{Internal Consistency of the MaSCS}

Calculations of the internal consistency of the two factors of the MaSCS revealed Cronbach's Alphas of $\alpha=.89$ for positive metacognitions about symptoms control and $\alpha=.88$ for negative metacognitions about symptoms control indicating good reliability. Table 2 shows Cronbach's Alphas if items were deleted and reveal that the internal consistency of both factors of the MaSCS would not be improved by removing any of the items.

\subsection{Correlation Analysis between the MaSCS, CFQ and SF-36 PF}

A series of Kolmogorov-Smirnov tests were conducted, suggesting that the distributions of all variables differed significantly from normality. As a result, a Spearman's Rho correlation matrix was generated (Table 3) showing that there were positive and significant relationships between both factors of the MaSCS and the CFQ. In addition, the matrix revealed that there was a significant and negative relationship between negative metacognitions about symptoms control and the SF-36 PF.

\subsection{Regression Analysis to Indentify Predictors of CFQ}

Several steps were taken to assess whether multicollinearity was present in the data. Firstly, the correlation matrix revealed no substantial correlations $(r>.9)$ between the predictor variables. Secondly, an inspection of the ranges of the Tolerance Index (TI; 0.41-0.95) and the Variance Inflation Factor (VIF; 1.052-2.439) for all predictor variables supported the absence of multicollinearity. Histograms and normality plots suggested that the residuals were normally 
distributed. Plots of the regression standardized residuals against the regression standardized predicted values suggested that the assumptions of linearity and homoscedascity were met. Additionally, the Durbin-Watson test suggested that the assumption of independent errors is tenable.

Table 4 presents a two-step hierarchical regression analysis with CFQ as the outcome variable. The predictor variables consisted of anxiety and depression on step 1 and both factors of the MaSCS on step 2. Results from this analysis indicated that both factors of the MaSCS contributed variance over and above anxiety and depression to CFQ scores. In the final step of the regression model both MaSCS factors and depression remained the only significant predictors of CFQ scores.

\section{Discussion}

In this study, we have presented the development and preliminary validation of an instrument aimed at assessing, in CFS patients, metacognitions about symptoms control in the form of symptom focusing and conceptual thinking about symptoms. This follows directly from earlier research that implicated metacognitions in CFS symptom severity (Lorraine Maher-Edwards et al., 2011) and research that has identified CFS-specific metacognitions (Maher-Edwards et al., 2012). The principal components analysis revealed that the MaSCS has two factors: positive and negative metacognitions about symptoms control. Correlation analyses also revealed significant and positive relationships between both of these factors and symptom severity, and a significant and negative relationship between negative metacognitions about symptoms control and physical functioning. Furthermore, the two factors of the MaSCS significantly predicted fatigue when controlling for both anxiety and depression. These findings are consistent with earlier studies implicating metacognitions in CFS (Maher-Edwards et al., 2012; Lorraine Maher-Edwards et al., 2011).

This study found that only negative metacognitions about symptoms control was significantly associated with physical functioning. Perhaps this is because physical functioning is secondary to the experience of fatigue symptoms in CFS: i.e. fatigue leads to avoidance, resulting in 
deconditioning and a reduction in physical functioning. If this mechanism is correct, it might explain why positive metacognitions about symptoms control were significantly associated with fatigue but not physical functioning.

Future studies might explore whether the MaSCS predicts, when controlling for negative emotion, levels of symptoms focus and conceptual thinking about symptoms. Further studies could also examine whether interventions designed to modify metacognitions can help to reduce levels of symptom severity by reducing the conceptual processing of symptoms. This might include interventions impacting on symptoms conceptual thinking such as rumination/worry postponement, detached mindfulness, and the challenging of metacognitions (Wells, 2008). It may also include techniques aimed at gaining greater attentional flexibility thus reducing symptoms focusing, such as the attentional training technique or situational attentional refocusing (Spada, Georgiou \& Wells, 2010; Wells, 2008).

The items from the MaSCS use the word 'symptoms' rather than specifically referring to fatigue. This was by design as the authors hope that this new instrument could be validated in other conditions, for example where physical symptoms persist in the absence of a causative diagnosis, or after successful treatment of a condition when such persistent symptoms can no longer be attributed to the condition.

This study is subject to several limitations that will have to be addressed by future research. First, social desirability, self-report biases, context effects, and poor recall may have contributed to errors in the self-report measurements. Second, the CFQ asks about a change in symptom severity over the previous month; it is plausible to assume that some patients may have been chronically ill for several years, entailing that a response to the item "no worse than usual" may still indicate severe experience of symptoms. This could affect the strength of the relationship between the newly developed instrument and levels of fatigue. Third, a cross-sectional design was adopted, and this does not allow causal inferences, and meant that the test-retest reliability of the new measure was not established. Fourthly, whilst anxiety and depression were controlled for, the presence of 
personality disorder was not. Studies have suggested that the there is a high prevalence of personality disorder in CFS (Henderson \& Tannock, 2004) and future studies that assess the clinical sensitivity of this newly developed self-report instrument will need to control for these constructs. Fifthly, this study utilizes self-report instrument to assess subjective experience and meta-awareness and as such, like much cognitive research, there is always doubt whether we are measuring the constructs we are intending to. Finally, the sample was moderate in size and many patients had received therapy. The 43 patients who had experienced CBT may have been exposed to the identification and exploration of cognitive constructs. However, standard CBT for CFS does not typically include the examination of metacognitions assessed here. This may be a stronger assumption because of the service from which participants were recruited. The service was one of the centres involved in the PACE trial (White et al., 2011) and many of its therapists were trained in the provision of protocol-driven manualized therapy whose sessions were often reviewed for treatment fidelity. Thus, the experience of treatment may not be particularly significant in explaining the current findings.

However, despite these limitations, we believe that the MaSCS is a useful preliminary instrument for future research that aims to more robustly establish the role of metacognitions in CFS as well as other conditions presenting with both explained and unexplained symptoms. The MaSCS may also help to deepen our understanding of the mediating role of symptom focus and conceptual thinking about symptoms in the experience of symptoms. 


\section{References}

Aggarwal, V. R., McBeth, J., Zakrzewska, J. M., Lunt, M., \& Macfarlane, G. J. (2006). The epidemiology of chronic syndromes that are frequently unexplained: do they have common associated factors? International Journal of Epidemiology, 35, 468-476.

Chalder, T., Berelowitz, G., Pawlikowska, T., Watts, L., Wessely, S., Wright, D., \& Wallace, E. P. (1993). Development of a fatigue scale. Journal of Psychosomatic Research, 37, 147-153.

Crawford, J. R., \& Henry, J. D. (2003). The Depression Anxiety Stress Scales (DASS): Normative data and latent structure in a large non-clinical sample. British Journal of Clinical Psychology, 42, 111-131.

Dimaggio, G., Procacci, M., Nicolò, G., Popolo, R., Semerari, A., Carcione, A., \& Lysaker, P. H. (2007). Poor metacognition in narcissistic and avoidant personality disorders: Analysis of four psychotherapy patients. Clinical Psychology and Psychotherapy, 14, 386-401.

Edmonds, M., McGuire, H., \& Price, J. (2004). Exercise therapy for chronic fatigue syndrome. Cochrane Database Systematic Review, 3.

Friedberg, F., Dechene, L., McKenzie Ii, M. J., \& Fontanetta, R. (2000). Symptom patterns in longduration chronic fatigue syndrome. Journal of Psychosomatic Research, 48, 59-68.

Henderson, M., \& Tannock, C. (2004). Objective assessment of personality disorder in chronic fatigue syndrome. Journal of Psychosomatic Research, 56, 251-254.

Henry, J. D., \& Crawford, J. R. (2005). The short-form version of the Depression Anxiety Stress Scales (DASS-21): Construct validity and normative data in a large non-clinical sample. British Journal of Clinical Psychology, 44, 227-239.

Hou, R., Moss-Morris, R., Bradley, B. P., Peveler, R., \& Mogg, K. (2008). Attentional bias towards health-threat information in chronic fatigue syndrome. Journal of Psychosomatic Research, 65, 47-50. 
Knoop, H., Bleijenberg, G., Gielissen, M. F. M., van der Meer, J. W. M., \& White, P. D. (2007). Is a Full Recovery Possible after Cognitive Behavioural Therapy for Chronic Fatigue Syndrome? Psychotherapy and Psychosomatics, 76, 171-176.

Komaroff, A. L., Fagioli, L. R., Geiger, A. M., Doolittle, T. H., Lee, J., Kornish, R. J., . . . Guerriero, R. T. (1996). An examination of the working case definition of chronic fatigue syndrome. The American Journal of Medicine, 100, 56-64.

Lovibond, P. F., \& Lovibond, S. H. (1995). The structure of negative emotional states: Comparison of the Depression Anxiety Stress Scales (DASS) with the Beck Depression and Anxiety Inventories. Behavior Research and Therapy, 33, 335-343.

Lysaker, P. H., Olesek, K., Buck, K., Leonhardt, B. L., Vohs, J., Ringer, J., . . Outcalt, J. (2014). Metacognitive mastery moderates the relationship of alexithymia with cluster C personality disorder traits in adults with substance use disorders. Addictive Behaviors, 39, 558-561.

Maher-Edwards, L., Fernie, B. A., Murphy, G., Nikčević, A. V., \& Spada, M. M. (2012). Metacognitive factors in chronic fatigue syndrome. Clinical Psychology \& Psychotherapy, 19, 552-557.

Maher-Edwards, L., Fernie, B. A., Murphy, G., Wells, A., \& Spada, M. M. (2011). Metacognitions and negative emotions as predictors of symptom severity in chronic fatigue syndrome. Journal of Psychosomatic Research, 70, 311-317.

Nisenbaum, R., Reyes, M., Unger, E. R., \& Reeves, W. C. (2004). Factor analysis of symptoms among subjects with unexplained chronic fatigue: What can we learn about chronic fatigue syndrome? Journal of Psychosomatic Research, 56, 171-178.

Price, J. R., Mitchell, E., Tidy, E., \& Hunot, V. (2008). Cognitive behaviour therapy for chronic fatigue syndrome in adults. Cochrane Database Systematic Review, 3. 
Sharpe, M. C., Archard, L. C., Banatvala, J. E., Borysiewicz, L. K., Clare, A. W., David, A., .. . et al. (1991). A report--chronic fatigue syndrome: guidelines for research. $J$ R Soc Med, 84(2), 118-121.

Sohl, S. J., \& Friedberg, F. (2008). Memory for fatigue in chronic fatigue syndrome: relationships to fatigue variability, catastrophizing, and negative affect. Behavioral Medicine, 34,29-38.

Spada, M. M., Georgiou, G., \& Wells, A. (2010). The relationship among metacognitions, attentional control and state anxiety. Cognitive Behaviour Therapy, 39, 64-71.

Ware, J. E., \& Sherbourne, C. D. (1992). The MOS 36-item short-form health survey (SF-36). I. Conceptual framework and item selection. Medical Care, 30, 473-483.

Ware, J. E., \& Kosinski, M. (2001). SF-36 Physical \& Mental Health Summary Scales: A Manual for Users of Version 1: QualityMetric.

Wells, A. (2008). Metacognitive Therapy for Anxiety and Depression. New York: The Guildford Press.

White, P. D., Goldsmith, K. A., Johnson, A. L., Potts, L., Walwyn, R., DeCesare, J. C., .. . group, P. t. m. (2011). Comparison of adaptive pacing therapy, cognitive behaviour therapy, graded exercise therapy, and specialist medical care for chronic fatigue syndrome (PACE): a randomised trial. Lancet, 377, 823-836.

White, P. D., Sharpe, M. C., Chalder, T., DeCesare, J. C., \& Walwyn, R. (2007). Protocol for the PACE trial: a randomised controlled trial of adaptive pacing, cognitive behaviour therapy, and graded exercise, as supplements to standardised specialist medical care versus standardised specialist medical care alone for patients with the chronic fatigue syndrome/myalgic encephalomyelitis or encephalopathy. BMC Neurolpgy, 7, 6. 
Table 1: Factor Loadings for the Revised Instrument

\begin{tabular}{lcc}
\hline & Factor 1 & Factor 2 \\
\hline When I experience symptoms, it's impossible to focus on anything else & .129 & $\mathbf{6 2 2}$ \\
If I focus on the symptom I can take the appropriate action to get better & $\mathbf{. 6 5 2}$ & -.297 \\
Ruminating about my symptoms helps me to figure out how to deal with them & $\mathbf{. 8 0 0}$ & -.047 \\
Thinking about my symptoms makes me feel frustrated & .032 & $\mathbf{. 7 3 7}$ \\
If I don't pay attention to my symptoms, I could push myself too far & $\mathbf{. 6 0 0}$ & .227 \\
Monitoring my symptoms helps me to predict how they will develop & $\mathbf{. 7 9 3}$ & .022 \\
Thinking about my symptoms makes me feel negative and down & -.092 & $\mathbf{. 8 8 5}$ \\
I monitor my symptoms so I can figure out my physical limitations &. $\mathbf{7 8 2}$ & -.044 \\
Thinking about my symptoms makes me feel exhausted & -.038 & $\mathbf{. 7 4 5}$ \\
Monitoring my symptoms enables me to better control them & $\mathbf{. 8 2 4}$ & -.019 \\
Focusing on my symptoms makes me feel anxious or stressed & -.125 & $\mathbf{. 8 0 2}$ \\
Monitoring my symptoms helps to keep me safe & $\mathbf{. 6 4 8}$ & .129 \\
I am not able to stop thinking about my symptoms once I start & .107 & $\mathbf{. 6 8 1}$ \\
By focusing on my symptoms I can detect when I am getting better & $\mathbf{. 7 5 3}$ & -.054 \\
Not paying attention to my symptoms could lead to my illness getting worse & $\mathbf{. 6 5 2}$ & .229 \\
Focusing on my symptoms makes me feel down & -.042 & $\mathbf{. 8 3 1}$ \\
Thinking about my symptoms could make them worse & .113 & $\mathbf{. 5 2 4}$ \\
\hline
\end{tabular}

Table 2: Corrected Item-Total Correlations and Cronbach’s Alphas if Item Deleted

\begin{tabular}{lcc}
\hline & $\begin{array}{c}\text { Corrected } \\
\text { Item-Total } \\
\text { Correlations }\end{array}$ & $\begin{array}{c}\text { Cronbach's } \\
\text { Alphas if } \\
\text { Item } \\
\text { Deleted }\end{array}$ \\
\hline Positive Metacognitions about Symptoms Control (Factor 1) & .486 & .889 \\
\hline If I focus on the symptom I can take the appropriate action to get better & .710 & .872 \\
Ruminating about my symptoms helps me to figure out how to deal with them & .568 & .883 \\
If I don't pay attention to my symptoms, I could push myself too far & .720 & .870 \\
Monitoring my symptoms helps me to predict how they will develop & .687 & .873 \\
I monitor my symptoms so I can figure out my physical limitations & .742 & .868 \\
Monitoring my symptoms enables me to better control them & .592 & .881 \\
Monitoring my symptoms helps to keep me safe & .651 & .876 \\
By focusing on my symptoms I can detect when I am getting better & .632 & .878 \\
Not paying attention to my symptoms could lead to my illness getting worse & & \\
\hline Negative Metacognitions about Symptoms Control (Factor 2) & .557 & .872 \\
\hline When I experience symptoms, it's impossible to focus on anything else & .634 & .865 \\
Thinking about my symptoms makes me feel frustrated & .799 & .847 \\
Thinking about my symptoms makes me feel negative and down & .642 & .864 \\
Thinking about my symptoms makes me feel exhausted & .675 \\
Focusing on my symptoms makes me feel anxious or stressed & .632 & .861 \\
I am not able to stop thinking about my symptoms once I start & .734 & .865 \\
Focusing on my symptoms makes me feel down & .471 & .881 \\
Thinking about my symptoms could make them worse & & \\
\hline
\end{tabular}


Table 3: Spearman’s Rho Correlation Matrix

\begin{tabular}{|c|c|c|c|c|c|c|c|c|}
\hline & Mean & SD & Range & $\begin{array}{l}\text { RAND SF- } \\
36 \text { PF }\end{array}$ & $\begin{array}{c}\text { DASS-21 } \\
\text { Depression }\end{array}$ & $\begin{array}{c}\text { DASS-21 } \\
\text { Anxiety }\end{array}$ & $\begin{array}{l}\text { MaSCS } \\
\text { PMSC }\end{array}$ & $\begin{array}{l}\text { MaSCS } \\
\text { NMSC }\end{array}$ \\
\hline CFQ & 41.5 & 10.1 & $14-56$ & $-.51 * *$ & $.52 * *$ & $.45^{* *}$ & $.24^{* *}$ & $.48^{* *}$ \\
\hline $\begin{array}{l}\text { RAND SF- } \\
36 \mathrm{PF}\end{array}$ & 51.2 & 24.7 & $0-100$ & & $-.41 * *$ & $-.38 * *$ & -.09 & $-.39 * *$ \\
\hline $\begin{array}{l}\text { DASS-21 } \\
\text { Depression }\end{array}$ & 7.9 & 6.5 & $0-21$ & & & $.71^{* *}$ & -.0 & $.56^{*}$ \\
\hline $\begin{array}{l}\text { DASS-21 } \\
\text { Anxiety }\end{array}$ & 5.7 & 5.2 & $0-21$ & & & & .13 & $.48^{* *}$ \\
\hline $\begin{array}{l}\text { MaSCS } \\
\text { PMSC }\end{array}$ & 23.3 & 6.5 & 9-36 & & & & & .12 \\
\hline $\begin{array}{l}\text { MaSCS } \\
\text { NMSC }\end{array}$ & 18.4 & 5.9 & 8-32 & & & & & \\
\hline
\end{tabular}

Table 4: Hierarchical Regression Model with CFQ as the outcome variable and DASS-21 and MaSCS as Predictor Variables.

\begin{tabular}{lllll}
\hline & $\boldsymbol{\beta}$ & $\mathbf{T}$ & $\boldsymbol{P}$ \\
\hline Step $\mathbf{1}$ & & & \\
\hline 1. DASS-21 Anxiety & 0.35 & 1.54 & 0.13 \\
\hline 2. DASS-21 Depression & 0.53 & 2.90 & 0.04 \\
\hline $\boldsymbol{r}^{2}=\mathbf{0 . 2 4}$ & & & & \\
\hline F Change=18.9 & & &
\end{tabular}

\section{Step 2}

\begin{tabular}{lllll}
\hline 1. DASS-21 Anxiety & 0.19 & 0.83 & 0.41 \\
\hline 2. DASS-21 Depression & 0.45 & 2.38 & 0.02 \\
\hline 3. MASCS PMSC & 0.25 & 1.99 & 0.05 \\
\hline 4. MASCS NMSC & 0.33 & 2.07 & 0.04 \\
\hline
\end{tabular}

$r^{2}=0.55$

\section{F Change $=4.8$}

$N=120,{ }^{*} p<0.05 ;{ }^{* *} p<0.01$.

PMSC $=$ Positive Metacognitions about Symptoms Control factor of the MaSCS.

NMSC = Negative Metacognitions about Symptoms Control factor of the MaSCS. 